\title{
Aspectos Mercadológicos dos Produtos não Madeireiros na Economia de Santarém-Pará, Brasil
}

\author{
Danielly Caroline Miléo Gonçalves ${ }^{1}$, João Ricardo de Vasconcellos Gama², \\ Francisco de Assis Oliveira ${ }^{1}$, Raimundo Cosme de Oliveira Junior ${ }^{3}$, \\ Giovânia Carvalho Araújo ${ }^{1}$, Larissa Santos de Almeida ${ }^{1}$ \\ ${ }^{1}$ Programa de Pós-Graduação em Ciências Florestais, Instituto de Ciências Agrárias, \\ Universidade Federal Rural da Amazônia - UFRA \\ ${ }^{2}$ Instituto de Biodiversidade e Floresta, Universidade Federal do Oeste do Pará - UFOPA \\ ${ }^{3}$ Núcleo de Apoio e Pesquisa e Transferência de Tecnologia - NAPT, Embrapa Amazônia Oriental
}

\begin{abstract}
RESUMO
Foram realizadas entrevistas semiestruturadas com comerciantes e vendedores informais da cidade de Santarém-PA de diferentes setores, relatando 107 produtos medicinais, que representam $14 \%$ do total dos produtos encontrados, incluindo plantas e produtos de origem animal. As espécies andiroba (Carapa guianensis Aubl.), copaíba (Copaifera reticulata Ducke) e cumaru (Dipteryx odorata (Aubl.) Willd.) são os produtos mais procurados pela população local. Os demais produtos somaram 92 , totalizando $86 \%$. A comercialização de artesanatos e biojoias é realizada no Centro de Santarém, em lojas especializadas, com mercadorias vindas de diversos lugares, inclusive de fora do Estado. Os principais consumidores são de classe média e alta, que valorizam a forma rústica e a importância socioeconômica que os produtos representam para as famílias que fabricam os artefatos.
\end{abstract}

Palavras-chave: produtos medicinais, artesanatos, biojoias.

\section{Market Aspects of non-timber Products in the Economy of Santarem, State of Para, Brazil}

\begin{abstract}
Semi-structured interviews were carried out with informal vendors and merchants from different sectors in Santarem, State of Para, reporting 107 medicinal products, which represented $14 \%$ of all products found, including plants and products of animal origin. The species andiroba (Carapa guianensis Aubl.), copaiba (Copaifera reticulata Ducke) and cumaru (Dipteryx odorata (Aubl) Willd.) are the most wanted products by the local population. The remaining 92 products account for $86 \%$ of the total. The sale of handicraft and biojewelry - coming from different places in and out of the state - take place in the city center at specialized stores. The main customers are people from the middle and upper classes who value the rustic form of the handicrafts and their socioeconomic importance for the producing families.
\end{abstract}

Keywords: medicinal products, handicraft, bio-jewelry. 


\section{INTRODUÇÃO}

A floresta Amazônica é fonte importante de recursos naturais para as atividades humanas, como a comercialização e o autoconsumo pelas comunidades locais. Desse modo, muitas comunidades ainda têm nela a fonte de alimento, renda, bem-estar social, saúde e segurança (Pastore Junior \& Borges, 1995; Food..., 2007). A prática ancestral, economicamente viável, de extração dos produtos não madeireiros, que proporciona estrutura e funcionalidade da floresta, tem surgido como um meio eficaz de harmonizar os papéis conflitantes das florestas tropicais (Balzon et al., 2004).

No entanto, a exploração desses produtos requer conhecimento prévio das espécies a serem coletadas, das técnicas que serão utilizadas nas colheitas desses produtos e seu rendimento real potencial para a comunidade beneficiada (Food..., 2007). Apesar de as comunidades possuírem conhecimento de algumas espécies florestais, ainda há vários entraves causados pelas limitações dos comunitários em coletar determinados produtos, tais como: a organização inexistente ou deficiente, que não possibilitaria a estruturação da produção para comercialização, e a ausência de políticas públicas, que dificulta a produção e a distribuição dos produtos oriundos das florestas até as feiras e os centros comerciais das cidades amazônicas (Allegretti, 1996).

No oeste do Pará, os principais produtos não madeireiros comercializados para fins medicinais são os óleos de copaíba, andiroba, pau-rosa e cumaru. Outros produtos comercializados são as biojoias confeccionadas com sementes, penas e fibras vegetais. Além das biojoias, os artesanatos são produzidos com recursos da floresta, como madeira, sementes, fibras vegetais e partes de animais. (Santana et al., 2008).

A maioria desses produtos comercializados no mercado, em estabelecimentos comerciais e feiras livres de Santarém, é proveniente da zona rural da cidade, sendo que uma pequena parte dos produtos vem de outros municípios, como Aveiro, Belterra, Monte Alegre e Oriximiná. Com base nessas informações, o objetivo do trabalho foi caracterizar os produtos florestais não madeireiros - para fins medicinais, de biojoias e artesanatos - comercializados na cidade de Santarém; com tais informações, podem-se definir quais produtos são essenciais para a população do município.

\section{MATERIAL E MÉTODOS}

\section{1. Área de estudo}

A cidade de Santarém $\left(2^{\circ} 24^{\prime} 52^{\prime \prime}\right.$ S e $54^{\circ} 42^{\prime}$ 36 " O) está situada na região oeste paraense, na meso região do Baixo Amazonas (Figura1), à margem direita do Rio Tapajós, na sua confluência com o rio Amazonas, distante cerca de $810 \mathrm{~km}$, em linha reta, da capital do Estado.

O clima é quente e úmido, com temperatura média anual variando de 25 a $28^{\circ} \mathrm{C}$. Apresenta pluviosidade média de $1.920 \mathrm{~mm}$. As temperaturas mais elevadas ocorrem nos meses de junho a novembro, e o período de maior precipitação pluviométrica é de dezembro a maio (Instituto..., 2009).

As principais atividades econômicas do município são: a extração de madeira, borracha e castanha-do-pará; as culturas de juta, mandioca, arroz, soja e milho; a criação de bovinos, suínos e aves de granja; a pesca e a indústria de fibras, além do processamento de pescado para exportação. Outra atividade econômica de grande crescimento é o turismo, que tem como atrações as praias, as cachoeiras, os lagos, as excursões ecológicas na mata e as numerosas tradições e festas folclóricas. São muito procurados pelos turistas os passeios de barco para acompanhar as linhas paralelas formadas pela diferença de densidades das águas do Rio Amazonas e do Rio Tapajós (Instituto..., 2009).

\subsection{Coleta de dados}

Foram realizadas entrevistas semiestruturadas com comerciantes e vendedores informais da cidade de Santarém, de diferentes setores, como: vendedores do mercado municipal, profissionais autônomos, comerciantes de artesanatos, farmácia homeopática e da COOMFLONA (Cooperativa Mista da FLONA Tapajós), que representa a associação que fabrica óleo de andiroba, copaíba e piquiá, bem como mel de abelha. 


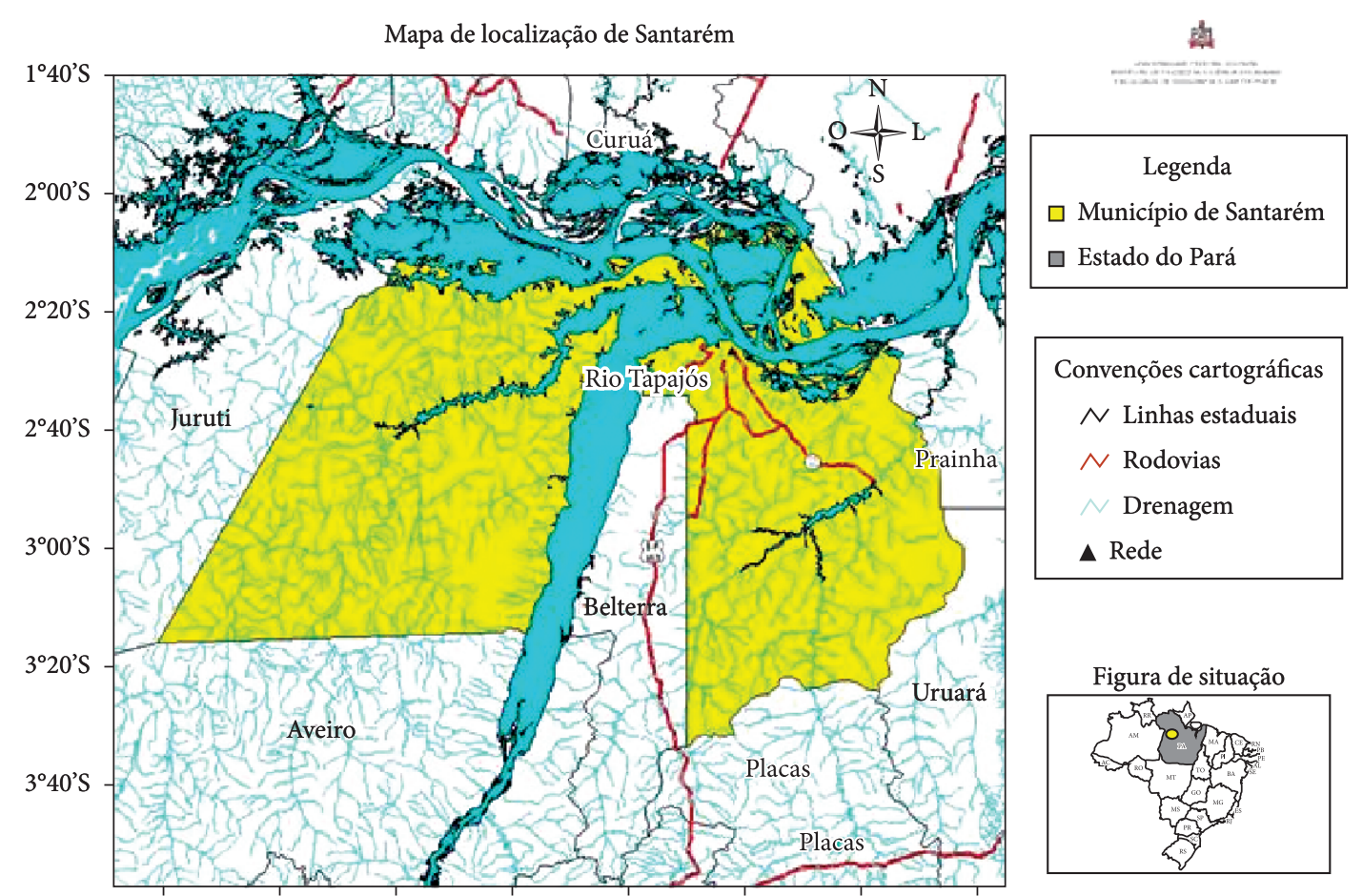

$56^{\circ} 20^{\prime} \mathrm{W} 56^{\circ} 00^{\prime} \mathrm{W} 55^{\circ} 40^{\prime} \mathrm{W} 55^{\circ} 20^{\prime} \mathrm{W} 55^{\circ} 00^{\prime} \mathrm{W} 54^{\circ} 40^{\prime} \mathrm{W} 54^{\circ} 20^{\prime} \mathrm{W} 54^{\circ} 00^{\prime} \mathrm{W}$

Figura 1. Mapa físico do município de Santarém-Pará, Brasil. Fonte: (Oliveira, 2008).

Figure 1. Physical map of the city of Santarem, Para, Brazil. Source: (Oliveira, 2008).

As entrevistas foram feitas por meio de um questionário aplicado aos donos de cada estabelecimento pesquisado, com perguntas referentes ao produto comercializado, à principal clientela $\mathrm{e}$ aos entraves que dificultam a comercialização dos produtos. Além das entrevistas, foram realizadas pesquisas bibliográficas, busca de informações em "sites" de organizações, como IBGE (Instituto Brasileiro de Geografia e Estatística) e FAO (Organização para Alimento e Agricultura). A pesquisa foi realizada nos meses de julho e agosto de 2010.

\section{RESULTADOS E DISCUSSÃO}

\subsection{Produtos não madeireiros para uso medicinal}

Os principais produtos não madeireiros usados para fins medicinais (Tabela 1) representam 14\% do total de 107 produtos encontrados, incluindo, além de plantas, produtos de origem animal.
Foram também enumeradas quantas vezes, em porcentagem, esses produtos foram citados pelos entrevistados. Observa-se que a andiroba (Carapa guianensis Aubl.) com 3,4\% das citações, a copaíba (Copaifera reticulata Ducke) com 3,4\% e o cumaru (Dipteryx odorata (Aubl.) Willd.) com 2,8\% são os produtos mais representativos, em função de sua importância medicinal reconhecida, além de terem uma grande procura por parte da população local. Os demais produtos somaram 92 e foram citados duas ou uma vez na pesquisa, totalizando $86 \%$.

Os preços expressos na Tabela 1 são baseados nas informações repassadas pelos comerciantes e, conforme esses dados foram extraídos, calculouse uma média de todos os preços de cada produto. Para fazer a valoração dos produtos, os comerciantes agregam ao valor a demanda dos mercados regional, nacional e até internacional. Alguns comerciantes não quiseram revelar os preços dos seus produtos comercializados, restringindo assim uma melhor conformidade dos dados amostrados. 
Tabela 1. Principais produtos de uso medicinal comercializados na cidade de Santarém-Pará, Brasil.

Table 1. Key pharmaceutical products marketed in the city of Santarem, Para, Brazil.

\begin{tabular}{|c|c|c|c|c|c|}
\hline Produtos & Nomes científicos & $\begin{array}{c}\text { Parte da } \\
\text { planta/animal }\end{array}$ & $\begin{array}{l}\text { Citações } \\
(\%)^{\star}\end{array}$ & $\begin{array}{l}\text { Unidade de } \\
\text { medida }\end{array}$ & $\begin{array}{l}\text { Preço médio } \\
\text { (R\$/unid.) }\end{array}$ \\
\hline Andiroba & Carapa guianensis Aubl. & Semente & 3,4 & $\mathrm{~L} / \mathrm{Kg}$ & 16,5 \\
\hline Copaíba & Copaifera reticulata Ducke & Seiva & 3,4 & $\mathrm{~L}$ & 15 \\
\hline Cumaru & Dipteryx odorata (Aubl.) Willd. & Semente/óleo & 2,8 & $\mathrm{~g} / \mathrm{kg}$ & 7,5 \\
\hline Mel de abelha & Mel & Mel & 2,2 & $\mathrm{~L}$ & 15 \\
\hline Noni & Morinda citrifolia $\mathrm{L}$. & Fruto & 2,2 & $\mathrm{~g}$ & $10,00^{\star * *}$ \\
\hline Quebra-pedra & Phyllanthus niruri L. & Folhas & 2,2 & Maço & 2 \\
\hline Verônica & Dalbergia subcymosa Ducke & Casca & 2,2 & $\mathrm{~kg}$ & 2 \\
\hline Amapá & Brosimum parinarioides Ducke & Seiva & 1,7 & $\mathrm{~L}$ & - \\
\hline Barbatimão & $\begin{array}{l}\text { Stryphnodendron pulcherrimum } \\
\text { (Willd) Hochr. }\end{array}$ & Casca & 1,7 & $\mathrm{~g}$ & - \\
\hline Carapanaúba & Aspidosperma auriculatum MG & Casca & 1,7 & g & - \\
\hline Eucalipto & Eucalyptus spp. & Folhas & 1,7 & $\mathrm{~g}$ & 2 \\
\hline Gergelim & Sesamum indicum L. & Semente/óleo & 1,7 & $\mathrm{~g} / \mathrm{L}$ & $40,00^{* * * *}$ \\
\hline Piquiá & Caryocar villosum Aubl. & Óleo & 1,7 & $\mathrm{~mL}$ & 5 \\
\hline Sacaca & Croton cajucara $\mathrm{L}$. & Casca & 1,7 & $\mathrm{~kg}$ & 3,5 \\
\hline Sucuúba & $\begin{array}{l}\text { Himatanthus sucuuba } \\
\text { (Spruce ex Muell. Arg.) Woodson }\end{array}$ & Seiva & 1,7 & $\mathrm{~L}$ & 7 \\
\hline
\end{tabular}

${ }^{*} \mathrm{n}^{\circ}$ de vezes citados nas entrevistas em $\%$; ${ }^{* *}$ preço referente à média dos preços para cada produto; ${ }^{* * *}$ valor correpondente a 100 g do fruto seco, vendido em pacotes no Mercado Municipal; ${ }^{* * *}$ valor referente a $1 \mathrm{~L}$.

O óleo de andiroba tem uma grande importância na vida dos povos da Amazônia. Até meados do século XX, a andiroba foi utilizada para a iluminação de casas em Belém e outras cidades do interior (Homma, 2008), mas seu principal uso é como remédio cicatrizante, repelente e na fabricação de produtos de higiene pessoal, tais como xampus, cremes e bálsamos (Shanley \& Medina, 2005).

Segundo Balzon et al. (2004), o óleo de copaíba apresenta propriedades medicinais, sendo empregado pela população local contra infecções, como cicatrizante e antisséptico das vias urinárias. Tem utilidade ainda na indústria de cosméticos e de vernizes. O óleo de copaíba apresenta uma grande heterogeneidade de cores, sendo necessária a escolha de espécimes de qualidade para efetuar sua domesticação (Santana et al., 2008).

As sementes do cumaru maceradas em água são utilizadas como antiespasmódico ${ }^{1}$, diaforético ${ }^{2}$ e contra problemas cardíacos e menstruais. Os frutos usados topicamente são eficazes no alívio da inflamação de ouvidos, sendo também indicado para tratar a pneumonia. O macerado dos frutos em ${ }^{1}$ Remédios que combatem contrações involuntárias causadas por alguma doença.

${ }^{2}$ Remédios que induzem a transpiração abundante; sudoríferos. álcool é usado contra cefaleia (Di Stasi e HirumaLima, 2002).

A maioria dos produtos mencionados na pesquisa não teve seu local de origem revelado, por causa do receio dos produtores de sofrer represálias por parte dos órgãos fiscalizadores, como IBAMA $^{3}$ e ANVISA ${ }^{4} .{ }^{4}$ No entanto, os vendedores que concordaram em falar relataram que a RESEX e a FLONA do Tapajós, nas comunidades de São Domingos, Maguari e Km 83 da Santarém-Cuiabá, fornecem para o mercado os óleos de andiroba, copaíba, piquiá e pupunha, o mel de abelha, o látex da seringueira, o leite de sucuúba, as folhas de vassourinha e a casca da verônica. Além da FLONA, as comunidades de Curuatinga, Vila Amazônica, Parintins, Arapixuna e Flexal extraem o óleo de andiroba vendido para farmácia de manipulação da cidade.

Outros produtos são oriundos de sítios nos bairros Jacamim, Jaderlândia e das comunidades do Planalto em Santarém, tais como: corama, cravo-dedefunto, escama de pirarucu, folha grossa, hortelã, manjericão, mastruz, patchouli e tucumã. O bairro do

${ }^{3}$ Instituto Brasileiro de Meio Ambiente e Recursos Naturais Renováveis.

${ }^{4}$ Agência Nacional de Vigilância Sanitária. 
Mararú fornece para as feiras: cana-mansa, crajirú, quebra-pedra, salva-de-marajó e vassourinha. Da vila Santa Maria, são fornecidos a erva-chumbo e o barbatimão. A vila de Alter-do-Chão e as cidades de Belterra, Alenquer e outras vizinhas, abastecem o mercado de Santarém com sementes de cumaru, que são exportadas para países da União Europeia, Estados Unidos e Japão.

Na FLONA Tapajós, os produtores de óleos de andiroba, copaíba, piquiá, e de mel de abelha têm uma representação comercial na cidade pela COOMFLONA, que, por sua vez, é responsável pelas embalagens e rotulação desses produtos. Essa cooperativa é responsável pela venda até o consumidor final, para alguns órgãos públicos ou negocia para exportação da matéria-prima para as empresas farmacêuticas internacionais. A extração dos óleos ainda é de forma artesanal, com uma unidade de beneficiamento de óleo.

Quarenta famílias distribuídas em cinco comunidades - São Domingos, Maguari, BR-163 Km 83, Pedreira e Nazaré - extraem e beneficiam a andiroba, a copaíba e o piquiá. Apesar de ter uma estrutura mais organizada em relação aos demais produtores, as principais limitações da comercialização do produto, segundo a cooperativa, é a falta de mão de obra, o que, por sua vez, influencia no preço final. Outro impasse é a ausência de legislação para os produtos não madeireiros, que dificulta a exportação da cooperativa para fora do País.

Existem várias dificuldades no comércio de produtos medicinais, dentre as quais a falta de certificação de controle de qualidade exigida pela ANVISA, pois a ausência do selo de qualidade restringe a venda dos óleos de andiroba e copaíba, e do mel de abelha por estabelecimentos comerciais, principalmente farmácias de manipulação e homeopáticas. Outros problemas relatados foram: adulteração por parte dos produtores, além de má conservação e falta de higiene. Esses impasses dificultam a competição desses produtos com os industrializados, como vem ocorrendo na maioria das farmácias de manipulação de Santarém, que utiliza óleos de andiroba e copaíba, e mel de abelha industrializados do Estado de São Paulo, por estes terem certificado de controle de qualidade.
Outros fatores limitantes na comercialização dos produtos não madeireiros, em especial o medicinal, segundo os entrevistados, é a falta de incentivos fiscais por parte do governo, para ajudar a expandir a extração. Por exemplo: os extratores muitas vezes não têm condições de arcar sozinhos com o transporte dos produtos até o centro da cidade para abastecer as lojas e feiras populares, de manter a extração contínua e a qualidade da produção dos produtos, e de possibilitar aumento da demanda de produtos para a exportação em outros mercados.

Para que as espécies medicinais alcancem o mercado externo, considerando-se que muitas delas possuem demanda de mercado, é preciso agregar valor, aumentar as etapas tecnológicas, melhorar a qualidade do produto, fazer extratos e preparados, e isolar princípios ativos (Castellani, 2002). Assim, além de desempenhar um papel importante na saúde das comunidades rurais, as plantas medicinais ocupariam um lugar privilegiado no mercado de medicamentos, com um potencial econômico que cresce na medida em que se conhecem os recursos e validam-se suas propriedades medicinais. Contudo, na maioria das vezes, esses produtos são comercializados na forma bruta, sendo os intermediários e as indústrias farmacêuticas os maiores beneficiados desse processo.

\subsection{Produtos não madeireiros comercializados em Santarém: artesanatos e biojoias}

A comercialização de artesanatos e biojoias é realizada no Centro de Santarém, em lojas especializadas, que compram diretamente os produtos dos artesãos. Os produtos comercializados são confeccionados com madeiras, como molongó, cedro, muiracatiara, fícus, jacarandá e pau-roxo, e sementes de açaí, bacaba, inajá, jarina, jutaí, lágrima, morototó, olho-de-boi, paxiúba, saboneteira, tento e tucumã (Tabela 2). Há também enfeites e utensílios confeccionados com cuias, ouriço de castanhado-pará, palhas de buriti, palha da costa, palha das palmeiras tucumã, piaçava e tururi, além do cipótitica, da seringa produzida a partir do látex da seringueira e de partes de animais, como escama de pirarucu, penas, ossos e dentes de boi e peixes empalhados, como acari, cujuba e piranha (Tabela 2). 
Tabela 2. Relação da matéria-prima, com o nome regional e científico, e os produtos para fins artesanais comercializados em Santarém-Pará, Brasil.

Table 2. Value of raw material, with the name and regional scientific and products produced for crafts sold in Santarem, Para, Brazil.

Matéria-prima

Produtos

Madeira

Cedro (Cedrela odorata Ruiz \& Pav., Meliaceae)

Fícus (Ficus nymphaefolia P. Miller, Moraceae)

Jacarandá-do-pará (Dalbergia spruceana, Papilonaceae)

Molongó (Malouetia tamaquarina (Aubl.) A. DC, Apocynaceae)

Muiracatiara (Astronium lecointei Ducke, Anacardiaceae)

Pau-roxo (Peltogyne confertiflora, Fabaceae)

Semente

Açaí (Euterpe oleraceae Mart., Arecaceae)

Bacaba (Oenocarpus distichus Mart., Arecaceae)

Castanha-do-pará (Bertholletia excelsa, Lecythidaceae)

Coco da bahia (Cocos nucifera, Arecaceae)

Inajá (Attalea maripa Aubl., Arecaceae)

Jarina (Phytelephas microcarpa, Arecaceae)

Jutaí (Hymenaea intermedia Ducke, Caesalpiniaceae)

Lágrima de nossa sra. (Coix lacryma -jobi L, Poaceae)

Morototó (Schefflera morototoni (Aubl) Dec \& Pl, Araliaceae)

Olho de boi (Mucuna altissima D.C., Fabaceae)

Paxiúba (Iriartea exorrhiza Mart., Arecaceae)

Saboneteira ( Sapondis saponaria, Sapindaceae)

Tento (Ormosia sp., Fabaceae)

Tucumã (Astrocaryum aculeatum G. Mey, Arecaceae)

Fibras

Buriti (Mauritia flexuosa, Arecaceae)

Cipó-titica (Heteropsis flexuosa, Araceae)

Palha da costa ( Raphia vinifera, Arecaceae)

Piaçava (Leopoldinia piassaba, Arecaceae)

Tucumã (Astrocaryum aculeatum, Arecaceae)

Tururi (Manicaria saccifera, Arecaceae)

Látex de Hevea brasiliensis

Forma de seringa (goma elástica)

Forma de balata (goma não-elástica)

Frutos

Castanha-do-pará (pixidios) ( Bertholletia excelsa, Lecythidaceae)

Cuia (exocarpo) (Crescentia cujete, Bignoniaceae)

Animais

Acari (Loricaria spp., Loricaridae)

Cujuba (Oxydoras niger, Doradidae)

Dente de boi (Bos taurus, Bovinae)

Dentes de peixes (diversas espécies)

Penas de galinha (Gallus gallus Phasianidae)

Penas de ganso (Branta sandvicensis, Anatidae)

Piranha (Serrasalmus rhombeu, Serrasalmidae)

Pirarucu-escama (Arapaima gigas, Osteoglossidae)
Porta-caneta, canetas, máscaras, baús, porta-chaves e quadros

Porta-caneta, canetas, máscaras, baús, porta-chaves e quadros

Porta-caneta, canetas, máscaras, baús, porta-chaves e quadros

Porta-caneta, canetas, máscaras, baús, porta-chaves e quadros

Porta-caneta, canetas, máscaras, baús, porta-chaves e quadros

Porta-caneta, canetas, máscaras, baús, porta-chaves e quadros 
Há variedade de produtos comercializados e estes têm origem em diversos lugares, inclusive de fora do Estado. Os principais artefatos vendidos são cestos, bolsas, bacias, chapéus e ventarolas de palha de tucumã, confeccionados pelas comunidades localizadas em Arapiuns, Lago Grande e Mararú; cestos, peneiras e tipiti ${ }^{5}$ de cipó-titica são produzidos pelos índios Uai-Uai, de Oriximiná no Rio Mapuera, e pela tribo dos Yanomamis; objetos de decoração, como quadros, porta-caneta, porta-chave, mascáras decorativas e baús de madeira são produzidos por diversos artesãos em Santarém; artefatos são produzidos com cuias da comunidade de Aritapera; objetos confeccionados com palhas de tururi, como bolsas, porta-níqueis e carteiras, são de Belém; a palha de piaçava é de Cachoeira Porteira e Rio Mapuera (Oriximiná); a palha do buriti ou linho de buriti usado na fabricação de bolsas e adereços vem do Maranhão; da seringa, é produzido o látex, com o qual se fabricam bolsas, sandálias, brinquedos e os artigos decorativos de balata (goma não elástica) são beneficiados na Comunidade de Maguari na FLONA, em Belterra, Santarém e Monte Alegre.

As bijuterias ou biojoias são confeccionadas com sementes de açaí, bacaba, castanha-do-pará (ouriço), coco-da-bahia, inajá, jarina, jutaí, lágrima, morototó, olho-de-boi, paxiúba, saboneteira, tento, tucumã (semente) e palha de tucumã, tendo origem de artesãos de Santarém (Alter-do-Chão, comunidades da Várzea e RESEX), como também de Oriximiná (Tribo Uai-Uai) e Manaus. Além dos artefatos produzidos com fibras vegetais, são comercializados produtos de origem animal, como penas, ossos e dentes polidos, que adornam os colares, pulseiras, brincos e anéis.

Os comerciantes atribuem preços aos produtos adicionando $30 \%$ no valor das peças adquiridas dos artesãos. $\mathrm{O}$ mercado de artesanatos e biojoias tem como consumidores homens e mulheres de classes média e alta, que valorizam a forma rústica com que são fabricados e tem a consciência da relação social e econômica que os produtos representam para as famílias que fabricam os artefatos. Em sua maioria, os principais consumidores são turistas nacionais (São Paulo, Rio de Janeiro, Paraná e Pernambuco), turistas internacionais (Estados Unidos, Japão, Inglaterra, ${ }^{5}$ Cesto, cilindro de palha, em que se põe a massa de mandioca
para ser espremida.
França, Espanha, Holanda e Alemanha), que viajam para conhecer a Amazônia em transatlânticos ou em pequenos grupos de mochileiros, aproveitam o passeio pela cidade e acabam adquirindo um souvenier, e a população local, que tem a cultura de usar as biojoias e o artesanato na decoração de suas casas.

Há lugares na cidade - como a Oficina Cabocla, composta por seis comunidades ribeirinhas do Rio Tapajós: Nova Vista, Nuquini, Surucuá, Pini, Prainha e Itapainuna - nos quais se fabricam móveis artesanais e peças artísticas para decoração a partir da madeira morta caída na floresta. As peças fabricadas são comercializadas na loja da cooperativa para os consumidores locais, regionais, nacionais e internacionais (turistas). Em Alter-do-Chão, as biojoias são fabricadas rusticamente e vendidas para os turistas que visitam a vila (Santana et al., 2008).

\section{CONSIDERAÇÕES FINAIS}

A dificuldade da organização dos atores (comerciantes, produtores, feirantes etc.) em associações e cooperativas está na aquisição da matéria-prima e na incrementação de novas técnicas de produção e exploração dos produtos. Se houvesse organizações democráticas e representativas, estas poderiam contribuir para a melhoria da condição tecnológica de produção, padronizando os recursos de acordo com que é exigido pelo mercado (melhor transporte dos produtos; locais adequados para o acondicionamento; maior visibilidade; capacitação de boas práticas de manuseio; confecções de embalagens, entre outros). A partir disto, se aumentaria a oferta de produtos de melhor qualidade, ampliando-se a produção para exportação.

\section{AGRADECIMENTOS}

Este trabalho foi realizado com o apoio da Iniciativa de Pesquisa em Saúde Mundial, uma associação dedicada ao financiamento de pesquisas entre os Institutos Canadenses de Pesquisa em Saúde, a Agência Canadense para o Desenvolvimento Internacional, o Ministério de Saúde do Canadá, o Centro Internacional de Pesquisas para o Desenvolvimento e a Agência de Saúde Pública do Canadá. 


\section{STATUS DA SUBMISSÃO}

Recebido: 14/05/2011

Aceito: 13/12/2011

Resumo publicado online: 17/12/2011

Artigo completo publicado: 31/03/2012

\section{AUTOR(ES) PARA CORRESPONDÊNCIA}

\section{Danielly Caroline Miléo Gonçalves}

Programa de Pós-Graduação em Ciências

Florestais, Instituto de Ciências Agrárias,

Universidade Federal Rural da Amazônica - UFRA

Av. Presidente Tancredo Neves, 2501, Montese,

CEP 66077-901, Belém, Pará, Brasil

e-mail: daniellycmg@gmail.com

\section{REFERENNCIAS}

Allegretti MH. Política para o uso dos recursos naturais renováveis: a região Amazônica e as atividades extrativistas. In: Clüsener-Goldt M, Sachs I. Extrativismo na Amazônia Brasileira: Perspectivas sobre o desenvolvimento regional. Paris: Compêndio Mab, UNESCO; 1996.94 p.

Balzon DR, Silva JCGL, Santos AJ. Aspectos mercadológicos de produtos não madeireiros - Análise retrospectiva [online]. Floresta 2004; 34(3):363-371. Available from: http://ojs.c3sl.ufpr.br/ojs2/index.php/ floresta/article/view/2422/2024.

Castellani DC. Critérios para o manejo sustentado de plantas medicinais em ecossistemas da Mata Atlântica [tese]. Viçosa: Universidade Federal de Viçosa; 2002.
Di Stasi LC, Hiruma-Lima CA. Plantas medicinais na Amazônia e na Mata Atlântica. 2nd ed. rev. e ampl. São Paulo: Editora UNESP; 2002. 604 p.

Food and Agriculture Organization of the United Nations - FAO. Non-wood forest products 13 [online]. Roma: Food and Agriculture Organization of the United Nations, 2007. [cited 2010 dez. 8]. Available from: http://www.fao.org/DOCREP/004/Y1457e/ Y1457e12.htm

Homma AKO. Extrativismo, biodiversidade $e$ biopirataria na Amazônia. Brasília: Embrapa Informação Tecnológica, 2008. 101 p.

Instituto Brasileiro de Geografia Estatística - IBGE. Produção Extrativismo vegetal e Silvicultural. Rio de Janeiro; 2009. v.23, 47 p.

Oliveira, J. M. G. C. de. Expansão urbana e periferização de Santarém-PA: Questões para o planejamento urbano. In: X Coloquio Internacional de Geocrítica: Diez años de cambio en el mundo, en la geografía y en las ciencias sociales, 1999-2008; 2008; Barcelona: universitat de Barcelona, 2008.

Pastore Junior F, Borges, V. Extração florestal não-madeireira na Amazônia: Armazenamento e comercialização. ITTO, FUNATURA, IBAMA, LATEQ-UnB; 1995. 73 p.

Santana AC, Silva IM, Oliveira CM, Silva RC, Filgueiras GC, Costa AD, et al. Caracterização do Mercado de produtos florestais madeireiros e não-madeireiros da Região Mamuru-Arapiuns. Belém: Fundação de Apoio á Pesquisa, Extensão e Ensino em Ciências Agrárias; 2008. 132 p.

Shanley P, Medina G. Frutíferas e Plantas Úteis na Vida Amazônica. Belém: CIFOR, Imazon, 2005. 300 p. 